\title{
A Trade-Investment Model for Distribution of Wealth
}

\author{
Nicola Scafetta ${ }^{a}$, Bruce J. West ${ }^{a, b}$ and Sergio Picozzi ${ }^{a}$ \\ ${ }^{a}$ Department of Physics and Free Electron Laser Laboratory, Duke University, \\ Durham, NC 27708, USA \\ b Mathematics Division, Army Research Office, Research Triangle Park, NC \\ 27709-2211, USA
}

\begin{abstract}
Econophysics provides a strategy for understanding the potential mechanisms underlying the anomalous distribution of wealth found in real societies. We present a computational nonlinear stochastic model for the distribution of wealth that depends upon three parameters and two mechanisms: trade and investment. To avoid economic paradoxes, the trade mechanism is assumed to be related to the poorer trader's wealth and to statistically advantage the poorer of the two traders. The two mechanisms together are shown to generate a distribution that reproduces the full range of the empirical wealth distribution, and not only the inverse power-law tail that Pareto found in western societies at the end of the 19th century.
\end{abstract}

Key words: Anomalous, condensation, interaction, Pareto, wealth PACS: 89.65.Gh, 87.23.Ge, 05.45.Df, 02.60.Cb

\section{Introduction}

The science of complexity leads to an understanding of complex physical phenomena through the construction of both equilibrium and dynamical models. These ideas have found application in engineering, biology, sociology and economics; specifically, in areas where phenomena have both the characteristics of randomness and determinism [1], present nonlinear and non-extensive behaviors and/or anomalous distributions. To understand the main mechanisms

\footnotetext{
* Corresponding author.

Email address: ns2002@duke. edu (Nicola Scafetta).
} 
that lead to the empirical distribution of wealth in a society is one of such applications.

More than a century ago the Italian sociologist and economist, Vilfredo Pareto determined that the cumulative probability of high income in western societies obeys to an inverse power law,

$$
P(w)=\int_{w}^{\infty} p(x) d x \propto \frac{1}{w^{\mu}},
$$

with $1 \leq \mu \leq 2[2]$ and where $p(x)$ is the probability density function (pdf) of wealth.

It is straightforward to observe that wealth and income are different concepts [3]. However, even if individual wealth and income are related in a very complex way, their empirical distributions, regarding an entire society, look similar. Compare, for example, the shape of the wealth distribution for United Kingdom studied in Ref. [4] with that of the income distributions for United Kingdom and other countries studied in Refs. [4,5]. Fig. 1 shows the cumulative distribution of wealth and income data for United Kingdom, downloaded from Inland Revenue, the British tax agency [6]. In particular, the reconstructed wealth distribution of the whole UK population was obtained by using an adjustment procedure applied to the data of all assets and liabilities of a person that must be reported at his or her death for the purpose of inheritance tax $[4]$.

Since Pareto's time a new field of research, econophysics, has emerged showing that physically-based models yield possible explanations of economic mechanisms in society [7]. In 1983 Montroll and Shlesinger [8] demonstrated that renormalization group scaling provides an interpretive context for Pareto's inverse power-law distribution. In fact, according to the renormalization group theory, a function $F(x)$ is homogeneous if there are two constants $a$ and $b$, such that $F(x)=a F(b x)$. The simplest solution to this equation is given by a Pareto law (1) with $\mu=\log a / \log b$ for $0<(a, b)<1$, [9]. Moreover, Montroll and Shlesinger argue that if $g(w)$ is the distribution of wealth excluding the very rich, the total distribution should have the anomalous form

$$
G(w)=g(w)+a G(b w)
$$

where $b$ is a mechanism that increases the variance of the distribution and $a$ is the probability of that mechanism being present in the society. For very large $w$ the distribution obtained from solving (2) is a Pareto's law

$$
\lim _{w \rightarrow \infty} G(w) \propto \frac{1}{w^{\mu}}
$$


and for low-medium level of wealth the distribution is

$$
\lim _{w \leftarrow \infty} G(w)=g(w) .
$$

Thus, they explain the inverse power-law tail in the distribution of wealth as a scaling effect in an equilibrium statistical society. On the other side, Eq. (2) suggests that the distribution of wealth at high and low ends is affected by different mechanisms. Indeed, full range empirical distributions of wealth or income [4,5] do present such a differentiation. For example, Fig. 1 shows the cumulative probability of wealth during 1996 and of income during 1998-1999 in United Kingdom [6]. It is easy to see the separation between low-medium level and high level that is characterized by a Pareto law and involves almost $1 \%$ of the population. In this paper we present a nonlinear physically-based model for wealth distribution able to interpret it.

While many have used variants of the Montroll-Shlesinger argument to explain several phenomena [10], here, we take a different approach and identify possible dynamical and stochastic mechanisms that lead to the observed distribution of wealth. We draw inspiration from the evolution equation postulated by Bouchaud and Mézard [11] that supposes distribution of wealth depends

on trades and investments, but we introduce different rules for trading dynamics. In Sec. 2 we review some models found in the econophysics literature like the mean-field approximation of the Bouchaud and Mézard model [11] and the kinetic theory approximation $[4,12,13,14]$ and explore some economic paradoxes induced by these approximations about the trade mechanism. In Sec. 3 we postulate a nonlinear stochastic trade-investment model that does not encounter the difficulties of the above approximations and we determine the properties of the model through numerical solutions. In Sec. 4 we argue the validity of our trade-investment model in interpreting the full range (both high and low ends) of the empirical wealth distribution as that recently found in the United Kingdom and shown in Fig. 1. Finally, we draw conclusions in Sec. 5 .

\section{Economic paradoxes of some asset exchange linear models}

Distribution of wealth depends mainly on two mechanisms: trade and investment $[4,5,11,12,13,14,15,16,17]$. We stress that in the physically-based models trade and investment should always be understood in a generalized sense. A linear model proposed by Bouchaud and Mezard [11], borrowed from the physics of directed polymers, describes the dynamics of the individual wealth 
$W_{i}(t)$ in a given a society of $N$ agents by mean of the following equation

$$
\frac{d W_{i}}{d t}=\eta_{i}(t) W_{i}+\sum_{j=1(\neq i)}^{N} J_{i j} W_{j}-\sum_{j=1(\neq i)}^{N} J_{j i} W_{i} .
$$

The component $\eta_{i}(t) W_{i}$ is a Gaussian multiplicative process with variance $\sigma$ that simulates the investment dynamics and it is related, for example, to the temporal change in the value of stocks $[11,18,19,20,21]$. The two sum terms of Eq. (5) describe the trade interaction network between the agent $i$ and all other agents in the society and $J_{i j}$ is the exchange rate between agents i and j.

Usually, Eq. (5) is solved with the simplifying assumption that the exchange rate for all agents is the same, i.e., $J_{i j} \equiv J / N$, so that Eq. (5) reduces to

$$
\frac{d W_{i}}{d t}=\eta_{i}(t) W_{i}+J\left(\bar{W}-W_{i}\right),
$$

where $\bar{W}=N^{-1} \sum_{i} W_{i}$ is the mean wealth. Eq. (6) is the mean-field approximation of the trade process [11]. Such an approximation is useful because Eq. (6) can be associated with a solvable Fokker-Planck equation with the following equilibrium pdf solution

$$
p_{e q}(w)=\Psi \exp \left[\frac{1-\mu}{w}\right] \frac{1}{w^{1+\mu}},
$$

where $\Psi=(\mu-1)^{\mu} / \Gamma[\mu]$ is the normalization constant and $\mu=1+J / \sigma^{2}$ is the Pareto exponent [11].

However, the power-law tail of Eq. (7) is due to the multiplicative term in (6) [22], and does not, by itself alone, assure the goodness of the mean-field approximation in describing a realistic trade process. In the absence of the multiplicative process, the mean-field approximation causes the wealth of all economic agents to exponentially converge toward the mean wealth $\bar{W}$. In fact, the solution of Eq. (6) without the multiplicative process is

$$
W_{i}(t)=\bar{W}+\left(W_{i}(0)-\bar{W}\right) \exp [-J t],
$$

implying that the trade dynamics has the effect of equalizing the wealth among all members of the society so that the observed condensation of the wealth is interpreted as due solely to the presence of the investment process.

This form of trade, implicit in the mean-field approximation, does not seem realistic because it assumes that the agent $i$ gives the fraction $J / N$ of his own 
wealth to the agent $j$ and, in exchange, receives the same fraction $J / N$ of agent $j$ 's wealth. So, a consistent amount of wealth, proportional to the rich agent's wealth, passes to the poor; an outcome that is not encountered in the real world. Instead, in a real trade, the rich always risk less than do the poor (in a statistical sense), because of their greater resources. This property should be fulfilled by the trade mechanism such that, in addition to the investment mechanism, the trade, too, has the capacity to produce a condensation of wealth.

A similar economic paradox is shared by the kinetic theory approximation when applied to the trade interaction between two agents $[4,12,13,14]$. This approximation yields a Maxwell-Boltzmann exponential distribution for energy $\left(p(E) \propto e^{-E / k T}\right)$. In fact, the kinetic theory approximation assumes that the transactions of wealth are similar to the transaction of energy in random elastic collisions between particles. Therefore, according to such an analogy, in a trade transaction the total wealth-energy (or a fraction of it according to the reaction scheme $\left[W_{i}, W_{j}\right] \rightarrow\left[\gamma W_{i}+\varepsilon(1-\gamma)\left(W_{i}+W_{j}\right), \gamma W_{j}+(1-\varepsilon)(1-\gamma)\left(W_{i}+W_{j}\right)\right]$, [14]) of both traders should mix, become randomly divided between the two traders and, on average, leave the two of them with the same amount of wealth. Also in this case, in a trade interaction between the rich and poor, the poor have an extraordinary chance to significantly increase their wealth; an unrealistic outcome. Moreover, the empirical distributions of wealth or income are not monotonic [6]; they increase, reach a maximum and, finally, decrease as an inverse power-law function. Therefore, the empirical distribution of wealth or income can not be recovered by the monotonically decreasing exponential distribution of Maxwell and Boltzmann.

It is also not convincing that in a trade a randomly selected agent loses a fixed fraction of his own wealth to a randomly selected winner $[12,15]$ according to the reaction scheme $\left[W_{i}, W_{j}\right] \rightarrow\left[W_{i}-\gamma W_{i}, W_{j}+\gamma W_{i}\right]$, where $0<\gamma<1$ is the fraction of wealth-energy of the loser gained by the winner. Such a trade mechanism would imply that in a transaction the richer the trader, the more he/she may lose in favor of the poorer trader.

These economic paradoxes, common to both the mean-field and kinetic theory approximations, seem to be related to the fact that those linear models assume a type of symmetric status of the two traders. Both approximations assume that the amount of wealth that can move from one agent to the other may be linearly related to the wealth of both agents or, at least, to the wealth of one of them randomly selected. We make what we believe to be a more realistic assumption regarding the common trade interaction dynamics. 


\section{A nonlinear trade-investment model}

We assume certain characteristics for the trading transaction that avoid the above paradoxes. First, we assume that in a trade between the rich and poor, the wealth that moves from one agent to the other is related to the wealth of the poorer of the two traders. In fact, only in a robbery the poorer trader can get a fraction of the wealth of the richer trader and the distribution of wealth of an entire society cannot reasonable be a product of a "theft-and-fraud" mechanism. Second, the trade mechanism has to take in account the role played by the prices in mediating exchange and how these prices emerge from ideal negotiations among agents that may belong to different social classes. Finally, we have to consider that a transaction of wealth in a trade is related to the difference between the price and the value of the asset.

We modify the linear Eq. (5) and suppose that the i-th agent's wealth $W_{i}(t)$ evolves according to the stochastic nonlinear equation

$$
W_{i}(t+1)=W_{i}(t)+r_{i} \xi(t) W_{i}(t)+\sum_{j=1(\neq i)}^{N} w_{i j}(t)
$$

Here, as in Eq. (5), the component $r_{i} \xi W_{i}$ is a Gaussian multiplicative process that simulates investment dynamics. The variable $\xi(t)$ is a Gaussian random variable and the standard deviation $r_{i}>0$ is the individual investment index. We interpreted $r_{i}=V \Pi_{i}$ where $V$ is the global investment index of the entire society, and $\Pi_{i}$ is the percentage of wealth $W_{i}$ that the $i$-th agent actually invests. The summation in Eq. (9) describes the trade transactions between agent $i$ and all other agents. If in the temporal interval $[t: t+1]$ two particular agents do not actually trade, there is no transfer of wealth between them, that is, $w_{i j}(t)=0$.

In a trade there is a flow of wealth between the two agents only if, in the case of the barter, the value of the two exchanged assets is different, or, in the case of the purchase, the value of the asset is different from the price paid for it. By expressing this concept in an equation, if the trader $i$ is the seller and the trader $j$ is the buyer, the exchanged wealth quantity $w_{i j}$ is given by

$$
w_{i j}=\text { price }_{\text {asset }}-\text { value }_{\text {asset }} .
$$

In fact, if the price and the value of the asset coincide, the trade would produce only a transfer of items and money from one agent to the other, but there would not be any transfer of wealth. Instead, if the seller (buyer) succeeds in selling (buying) an item for a price that is higher (lower) than the actual value of the item, the seller (buyer) gains wealth from the buyer (seller). Therefore, 
the trade interaction between two agents is assumed to follow the scheme $\left[W_{i}(t), W_{j}(t)\right] \rightarrow\left[W_{i}(t+\delta t), W_{j}(t+\delta t)\right]$, where

$$
\left[W_{i}(t+\delta t), W_{j}(t+\delta t)\right]=\left[W_{i}(t)+w_{i j}(t), W_{j}(t)+w_{j i}(t)\right] .
$$

Because of Eq. (10), the trade variable $w_{i j}$ is antisymmetric $\left(w_{i j}=-w_{j i}\right)$, may be positive or negative and indicates the amount of wealth that moves from one agent to the other in each trade. The antisymmetric nature of the trade variable $w_{i j}$ implies that the trade itself, contrary to the investment process, conserves the total wealth because it can only move wealth from one agent to another, but can neither create nor destroy wealth.

The amount of wealth $w_{i j}$ that may move from the trader $i$ to the trader $j$ is a stochastic nonlinear variable that has to be a fraction of the wealth of the poorer of the two agents and depends nonlinearly on the wealths of both traders. The simplest hypothesis consistent with these assumptions is to suppose that $w_{i j}$ is a Gaussian variable with density

$$
p\left(w_{i j}\right)=\frac{1}{\sigma \sqrt{2 \pi}} \exp \left[-\frac{\left(w_{i j}-\bar{w}_{i j}\right)^{2}}{2 \sigma^{2}}\right],
$$

where $\bar{w}_{i j}$ is the mean wealth that may move between the two traders $i$ and $j$,

$$
\sigma=h W_{i j}
$$

is the standard deviation of the distribution of $w_{i j}$ where $0<h<1$ indicates the fraction of $W_{i j}$ that may be involved in the trade, and the quantity

$$
W_{i j}=W_{j i}=\min \left(W_{i}, W_{j}\right)
$$

is the lesser of the two agents' wealth. We refer to the index $h>0$ as the poverty index. In fact, in a poor society the ratio of cost to wealth is higher and, therefore, the higher is the amount of wealth that may move in a singletrade transaction. The choice of the expression that indicates the mean wealth $\bar{w}_{i j}$ that may move from the trader $i$ to the trader $j$ is of crucial importance. For reasons explained subsequently, we assume that

$$
\bar{w}_{i j}=\alpha_{i j} h W_{i j}
$$

where the variable $\alpha_{i j}$ is given by a nonlinear expression depending on the 
wealths of both traders, for example,

$$
\alpha_{i j}=f \frac{W_{j}-W_{i}}{W_{j}+W_{i}} .
$$

We refer to the index $f>0$ as the social index because it provides an advantage to the poorer of the two traders. In fact, by using Eqs. (12), (15) and (16) it is easy to prove that if the wealths $W_{i}$ and $W_{j}$ are almost the same, then $\alpha_{i j} \approx 0$ and both traders have an equal chance of doing either a good or bad deal. If, instead, for example, $W_{j} \gg W_{i}$, we have $\alpha_{i j} \approx f$, the distribution $p\left(w_{i j}\right)$ is shifted toward positive values and the trader $i$, that in this example would be the poorer, has a better chance to do a good deal and increase his own wealth. Finally, the amount of moving wealth is always a fraction of the wealth of the poorer of the two agents.

A possible interpretation of the trade interaction mechanisms described by Eqs. (10), (11) and (12) may be formulated by considering that in a trade there is a transfer of wealth between two agents only if the value of the asset is different from the price paid for it, Eq. (10). The variable $w_{i j}$ of Eqs. (10), (11) and (12) measures such a difference and is stochastic because the price of an asset, or similar assets, is not unique but varies within a range in a real society. So, there may be the possibility to buy or sell an asset at a price that may be lower or higher than the value of that asset. We observe that this price dispersion is due to the fact that the price is an agreement between the two traders that follows an explicit or implicit trade negotiation and different negotiations may yield different prices for the same item. Therefore, we are not assuming the "law of one price" [23], which is a simplistic assumption made in most economic models (including Pareto's [24]) that attempt to dynamically address the problem. On the contrary, the value of an item is a concept that involves the entire society and the total wealth of an individual is measured by the total value of his or her belonging and not by the price of such possessions.

The stochastic nonlinear bias, see Eqs. (15) and (16), that favors the poorer trader is due to the fact that a trade can take place only if the two agents, through negotiation, reach an agreement about the price of the asset. The trade transaction has a higher probability to occur if the price is below a threshold at which the buyer would like to buy. Because this threshold increases with the total wealth of an agent, when a wealthy agent would like to buy something from a poorer agent, there is a higher probability that the transaction occurs at a higher price than when a wealthy agent would like to sell the same item to a poorer agent. Alternatively, we may say that the poor are constrained by their poverty to be more careful in their trades and therefore, for example, they may often look for the best price opportunity for saving money. On the other hand, because of their economic strength, the rich may be willing to pay a premium to purchase items. This asymmetric 
disadvantage-advantage tends to disappear when the two traders are economically equivalently.

In the next subsections we investigate step by step the properties of the tradeinvestment model. Subsections 3.1 and 3.2 are devoted to study the properties of the trade interaction without any investment.

\subsection{Symmetric-chance trade-alone model}

Let us assume $h>0, f=0$ and $r=0$, that is, we have a trade-alone economy according to which both agents have the same chance to gain or lose and in which there are no investments. We observe that $f=0$ implies $\bar{w}_{i j}=0$ in Eq. (12), therefore, the distribution $p\left(w_{i j}\right)(12)$ is symmetric and centered on zero. In this model it is easy to prove that almost all the wealth of our ideal society concentrates in the hands of a few agents. In fact, because the wealth $w_{i j}$ is related to the poorer agent's wealth, through the standard deviation $\sigma=h W_{i j}$ of the probability distribution (12), the risk for the rich trader is smaller because if he/she loses a certain amount of wealth in a tradetransaction, the loss is a smaller fraction of his/her own wealth than that which the poorer agent may lose. Consequently, there is a high probability in this model that few people accumulate almost the entire wealth available and the others become devastatingly poor.

Fig. 2 shows the huge wealth gap between the rich and poor produced by the symmetric-chance model. This gap increases with the number of trade interactions and the process is boosted by increasing the poverty index $h$. In fact, in such an eventuality the poor may lose a larger portion of their wealth in each single transaction. If the model is implemented with a wealth threshold below which an agent is considered economically impotent, the entire wealth of the society will concentrate in the hands of one person while all the others die.

This economic catastrophe, the gambler's ruin problem [25], is due simply to the intrinsic economic strength of the rich and, therefore, such a collapse may happen also without any economic abuse or exploitation of the poor by the rich. This condensation aspect of the trade-interaction dynamics is completely masked by the mean-field approximation of the Bouchaud and Mezard's model [11] that, without any investment, yields a uniform distribution of wealth. Moreover, the strong wealth condensation of the fair trade economy also explains why, to avoid economic collapse, in real societies there exist a number of mechanisms that have the effect of redistributing wealth by advantaging the poor. 


\subsection{Asymmetric-chance trade-alone model}

The redistribution mechanism that advantages the poor is implemented by supposing that the probability distribution of transaction of wealth $w_{i j}$, Eq. (12), is biased in favor of the poorer trader. The bias is introduced through the mean transaction wealth $\bar{w}_{i j} \neq 0$ because we now assume the social index $f>0$ (16), and we again assume $h>0$ and $r=0$. A social index $f>0$ provides an advantage to the poorer of the two traders.

The asymmetric-chance model redistributes the wealth, as shown in Fig. 3, and leads to a stable distribution. In addition, this model shows the emergence of a large middle class, followed by a smaller poor class and an even smaller rich class. The economic gap between the richest and poorest is not unrealistically wide, as it was in the symmetric-chance model. The condensation of wealth, that is, the wealth separation between the richest and poorest increases by decreasing the social index $f$. Fig. 3 also shows that this type of distribution of wealth can be well fitted with a Gamma-like distribution

$$
p(w)=a(w-c)^{\eta} \exp (-d w)
$$

that has an exponential-like tail. In the computer simulation we suppose a society of 100,000 economical agents with an initial uniform distribution of wealth and determine the wealth pdf after 100 million random trade-interactions between two randomly selected agents.

Fig. 4 shows how the asymmetric-chance model depends on the poverty index $h$. By keeping the social index $f$ fixed, the wealth gap between the richest and poorest increases by increasing the poverty index $h$. In fact, in a rich society, one based on trade alone, wealth is better distributed because it is more difficult for wealth to condense into the hands of a very few agents because people need less money to buy items. Consequently, in each tradeinteraction agents may lose a smaller fraction of their wealth.

In summary, our trade interaction model is based on the balance of two opposing contributions: (i) the condensation mechanism of the fair trade dynamics; (ii) a redistribution factor giving a stochastic advantage to the poor. When both processes are present, the trade interaction mechanism produces a stable distribution of wealth well fitted with a Gamma-like distribution. 


\subsection{Pareto tail in the trade-investment economy}

In the last step we assume all three indexes of the trade-investment model are positive: $h>0, f>0$ and $r>0$. Fig. 5 shows the distribution of wealth for three computer simulations in which we implement the previous model by assuming that every 10,000 trades the wealth of all agents is re-initialized by the multiplicative process. We fit the tail of the computer-generated wealth pdf with a Pareto distribution $1 / x^{\delta}$, where $\mu=\delta-1$ is the Pareto's exponent. These computer-generated wealth pdfs may be apparently well-fitted by functions of the form

$$
p(w)=a w^{\gamma} /(1+b w)^{\gamma+\delta} .
$$

However, we stress that in the absence of the analytic solution to Eq. (9), we do not know the exact analytic form for these computational distributions and, as we will explain in Sec. IV, the true solution to Eq. (9) is more complex than Eq. (18). However, we notice that the functions (17) and (18) may be related, one the other, in the limit $r \rightarrow 0$ because an exponential function can be obtained from a power-law function via an appropriate limit procedure $[26]$.

Fig. 5 shows that wealth condensation increases by increasing the investment index $r$ and by decreasing the social index $f$. The connection with the poverty index $h$ is more complicated and will be explained in the next section. As in the asymmetric-chance model, we obtain a stable distribution of wealth $p(w)$ that partitions society into three classes. Fig. 6 shows the cumulative probability $P(w)$ for an economy with $f=0.3, h=0.05$ and $r=0.075$, fitted with the Pareto distribution having an index $\alpha=\delta-1=1.5$. This figure is consistent with the fit to U.S. income data made in Figure 35 of Montroll and Badger [27]. According to a more recent study made by Drăgulescu and Yakovenko [4] concerning the cumulative probability of income in the United Kingdom and the United States, during the period 1994-1999 the United Kingdom was characterized by a Pareto exponent in the interval $1.8<\alpha<2.3$ and during the year 1998 the United States was characterized by a Pareto exponent $\alpha \approx 1.7$. By assuming that the cumulative wealth distributions have Pareto exponents similar to that of the correspondent income distributions, these different values of the Pareto exponent would imply that wealth is more concentrated in the United States than it is in the United Kingdom. By supposing similar investment economies of the two countries, the higher value of the UK Pareto's index can be interpreted in the context of our model as being due to the social policies adopted in the UK, yielding a higher social index $f$.

The inset in Fig. 6 shows that in the wealth interval $[10,100]$ the cumulative probability $P(w)$, for an ideal economy with $f=0.3, h=0.05$ and $r=$ 
0.075, can be fitted by an exponential function. A similar result is found by Drăgulescu and Yakovenko [4] in empirical cumulative probability of wealth in the United Kingdom. However, the exponential fit, that would justify the application of the Maxwell-Boltzmann distribution model, see Sec. 2, to the wealth as well as income distribution at low value, is here only apparent and not real. These authors recognize that at low income, and we suppose at low wealth as well, the pdf is not monotonic because it increases and then decreases, and may be better fit by a Gamma distribution, but Drăgulescu et al. [4] do not have any model to reproduce this behavior of the empirical data. Instead, as Fig. 5 shows, this non-monotonic behavior of the distribution at low and middle wealth value is well reproduced by the trade-investment model. Therefore, caution must be exercised in drawing conclusion from the data fitting of the cumulative distribution alone.

\section{Wealth and income distributions in societies}

As anticipated in the introduction and in the previous section the full range empirical distributions of income of several countries $[4,5,8,28]$ present a differentiation between the rich and non-rich classes. Fig. 1 shows that, at least for United Kingdom, wealth and income distributions look very similar, therefore we suppose that such a differentiation is a general property for the wealth distributions as well. The middle-low wealth range appears to follow a distribution that Drăgulescu et al. [4] fit with an exponential while Souma, following Gibrat [29], fits with a log-normal distribution. The higher wealth range, that involves only $1-2 \%$ of the population, maintains a Pareto tail. This particular anomalous shape may be recovered by the trade-investment model as Figs. 7 and 8 show.

A possible interpretation of the separation effect is that the investment index varies among the members of society, as we have already suggested in Eq. (9). The simplest option is to assume that $N_{1}$ agents invest their wealth with a investment index $r_{1}$ and $N_{2}=N-N_{1}$ agents invest their wealth with a different investment index $r_{2}$ :

$$
\begin{aligned}
& \frac{d W_{i}}{d t}=r_{1} \xi W_{i}+\sum_{j=1(\neq i)}^{N} w_{i j} \quad, \quad i \leq N_{1} \\
& \frac{d W_{i}}{d t}=r_{2} \xi W_{i}+\sum_{j=1(\neq i)}^{N} w_{i j} \quad, \quad i>N_{1} .
\end{aligned}
$$

In the eventuality that the system is characterized by more than two invest-

ment indexes, we have simply to expand the set of equations to accommodate 
them. In theory, the investment index $r$ may change for each trader. Fig. 7 shows two cumulative distributions of our ideal economic society of 100,000 agents. The social index is $f=0.3$, the poverty index is $h=0.05$ and the investment index is $r_{1}=0.075$ or $r_{1}=0.055$ for 50,000 agents and $r_{2}=0$ for the other 50,000 agents. The curves look very similar to the empirical distributions found in Refs. [4] and [5], and in Fig. 1. The Pareto exponents are $\alpha=1.5 \pm 0.02$ and $\alpha=2.5 \pm 0.02$, which are in the range the empirical distributions.

However, Fig. 7 shows that the Pareto law characterize only $1 \%$ of the population, while in the simulation we assumed that $50 \%$ of the population is characterized by a trade-investment economy and the other $50 \%$ by a tradealone economy. This fact suggests that the multiplicative process of the tradeinvestment economy is effective only for a small portion of the population. In fact, Fig. 8 suggests a slightly different and deeper interpretation of the dynamics of the trade-investment model. Fig. 8 shows the cumulative probability of two trade-investment economies with two different poverty index $h=0.03$ and $h=0.09$. The investment index $r=0.075$ and the social index $f=0.3$ are the same for all members of the society, so the computer simulation is done by using only one kind of evolution equation Eq. (9).

In fact, the explanation of Fig. 8 is quiet suggestive. The society may be divided in two groups: the small rich class, almost $1 \%$ of the population, and all the others. The difference between the two groups is in the economic mechanism that has the greatest effect on the wealth of each group. The meaning of the results shown in Figs. 7 and 8 is that the change of wealth of the rich class is more influenced by the investment economy while the wealth of all the others is more influenced by the trade economy. In fact, for people that are not rich the amount of wealth that they may gain or lose with some investment is compatible or lower than what they may gain or lose in trades. Instead, for the rich, trade alone may move only a small portion of their wealth because most of their trades involve agents poorer than themselves. So, for the rich, the investment part of the economy dominates, and because the investment economy is described by a multiplicative process, the wealth of the rich follows the Pareto inverse power law. For the other side, by increasing the poverty index, the capacity of the trade to move wealth is amplified and this explains how by increasing the poverty index $h$, the difference between the rich (that are characterized by the investment economy that generates a Pareto's wealth distribution), and all the others (that are characterized by the trade economy that generates a Gamma-like wealth distribution) becomes more prominent, as shown in Fig. 8.

In summary, the distribution of wealth shows an anomalous shape well described by a Gamma distribution at low and middle wealth and a Pareto's tail 
at high wealth. This complex shape may be approximately described by

$$
p(w)=a w^{\gamma}\left[\exp (-b w)+\frac{1}{(1+c w)^{\gamma+\delta}}\right] .
$$

that is a pdf of the type suggested by Montroll and Shlesinger, Eq. (2), and depends on many parameters that characterize the difference between the trade and the investment economy. The Pareto index increases by increasing the social index $f$ or by decreasing the investment index $r$. The dependency of the wealth condensation phenomenon on the poverty index $h$ seems more complex. In fact, in Sec. III we determined that wealth condensation in a trade-alone economy increases by increasing the poverty index $h$. However, Fig. 8 shows that in a trade-investment economy, wealth condensation may also decrease by increasing the poverty index $h$. Therefore, wealth condensation is a non-monotonic function of $h$. We stress that it is necessary to have a valid trade-interaction model to obtain all the above results. We have shown that by changing the value of the three indexes, our trade-interaction model is able to give a consistent explanation of the observed distribution of wealth.

\section{Conclusion}

While some linear asset exchange models like those based on the mean-field and kinetic theory approximations may present serious economical paradoxes, the science of complexity and physically-based models may still yield possible explanations of the distribution of wealth in a society. The nonlinear trade-investment model determines the main mechanisms necessary to obtain a stable three-class society with a Pareto distribution for high incomes. The mechanisms present in the trade-investment model are an asymmetric trade interaction that statistically favors the less wealthy of the two traders involved in the trade and a multiplicative stochastic investment process. A symmetric trade-alone mechanism would yield a strong wealth condensation phenomenon and therefore an unstable society. Both mechanisms are necessary to overcome the wealth condensation effect and stabilize the society. The detailed nature of the relative contribution of the two mechanisms are determined by the three control parameters: the social index $f$, the investment index $r$ and the poverty index $h$.

Here, we cannot do a detailed economic analysis of the mechanisms that advantage the poor in society by inhibiting the transfer of the entire wealth to the rich. In general, the richer party is less risk averse when bargaining over a given amount than is the poorer party, therefore the poorer party should be a stronger bargainer than the rich to get the better of the deal. In particular, we 
can consider the tendency of the poor to look for the best price opportunity for most single items for saving money, the tendency of the prices to increase in places where the customers are supposed wealthy, the employee rights that advantage workers by mean of adequate salary policies, the graduated income and luxury tax policies that require the rich to pay a higher percentage of taxes than is paid by the wage earner, and many others. In the absence of such social and psychological pro-poor mechanisms it is easy to envision that the society would collapse because the entire wealth would condense into the hands of a very few people. Also we observe that the above mechanisms can be interpreted as a kind of generalization of the price discrimination in monopoly theory [30] applied to the social classes of an entire society.

Investment is the dominant economic tool of the very rich. Montroll and Shlesinger [8] argued that the rich have economic mechanisms available to them that the ordinary citizen does not, and these mechanisms provide the amplification that generates the inverse power law distribution. The tradeinvestment model incorporates this amplification through the multiplicative form of the investment term in the dynamic equation. The magnitude of the amplification factor is determined by the investment index $r$.

Trade is the dominant economic tool of the majority of society. Salaries and taxes are considered particular trades. In the upper economic limit of the middle class some individuals increase their wealth as much through investment as they do through trade, providing a transition from a Gamma-like distribution that characterizes a trade-dominated economy to a Pareto's tail distribution produced by the investment economy. What distinguishes the trade-investment model from other models that have been proposed is the recognition that in order for a society to be stable, rather than the poor being exploited in trades with the rich they must have an advantage, at least in a statistical sense. This statistical advantage acts to narrow the gap in wealth between the richest and poorest members of society. The two parameters that control this narrowing of the gap is the social index $f$, that enables a poor individual to optimize his/her return in a trade with the rich, and the poverty index $h$, that determines the fraction of the poorer agent's wealth that can be transferred in any given transaction.

When we implement the trade-investment model with the three indices $f, h$ and $r$, the wealth pdf assumes the inverse power-law distribution of Pareto at the high wealth end, and, more realistically, still retains a small but finite population at the low wealth end. Moreover, the cumulative distribution of wealth assumes the shapes depicted in Figs. 7 and 8 that are similar to the shape depicted in Fig. 1, in which the rich class is distinguished by following Pareto's inverse power law and the cumulative distribution of wealth for the remainder of society is apparently well fit by a Gamma distribution. Numerical simulations shown that the dynamics of the trade-investment model Eq. (9) 
reproduces the shape of the universal structure of the cumulative distribution of wealth that is assumed to be similar to the cumulative distributions of income obtained for phenomenological data from around the world.

\section{Acknowledgment:}

N.S. thanks the Army Research Office for support under grant DAAG5598D0002. We would like to thank prof. M. Boianovsky and prof. T. Groves for some useful discussion.

\section{References}

[1] B.J. West, Physiology, Promiscuity and Prophecy at the Millenium: A Tale of Tails, World Scientific, Singapore (1999).

[2] V. Pareto, Manuale di economia politica, Milano, Società Editrice, 1906. V. Pareto, Cours d'Economie Politique, Lausanne and Paris (1897).

[3] J. Black, Dictionary of Economics, Oxford University Press, New York (2002).

[4] A. Drăgulescu and V. M. Yakovenko, "Exponential and power-law probability distributions of wealth and income in the United Kingdom and the United States," Physica A, 299, 213-221 (2001).

[5] W. Souma, "Universal structure of the personal income distribution," Fractals, 9 No. 4, 463-470 (2001).

[6] http://www.inlandrevenue.gov.uk/stats/: Inland Revenue, National Statisics, UK.

[7] H. E. Stanley and R. N. Mantegna, in An introduction to econophysics, Cambridge university press, Cambridge UK (2000).

[8] E.W. Montroll and M.F. Shlesinger, "Maximum-entropy formalism, fractals, scaling phenomena, and 1/f noise- a tale of tails," J. Stat. Phys. 32, 209 (1983).

[9] H.E. Stanley, Introduction to Phase Transitions and Critical Phenomena, Oxford University Press, New York (1971).

[10] B. Gutenberg and C.F. Richter, "Frequency of earthquakes in California" Bull. Seismol. Soc. Am. 34, 184 (1944); J.M. Carlson, J.S. Langer and B.E. Shaw, "Dynamics of earthquake faults," Rev. Mod. Phys. 66, 657 (1994). O. Peters, C. Hertlein and K. Christensen, "A complexity view of rainfall," Phys. Rev. Lett. 88, 018701 (2002). C.K. Peng, J. Mietus, J.M. Hausdorff, S. Havlin, H.G. Stanley and A.L. Goldberger, "Long-range anticorrelations and nongaussian behavior of the heartbeat," Phys. Rev. Lett. 70, 1343 (1993). B.J. West, R. Zhang, A.W. Sanders, S. Miliyar, J. H. Zucherman and B. Levine, "Fractal fluctuations in cardiac time series," Physica A 270, 552 (1999). B.J. West, V. Bhargava and A.L. Goldberger, "Beyond the Principle of Similtude: 
Renormalization in the Bronchial Tree" J. Appl. Physiol. 60 3, 1089-1097 (1986). P. Grigolini, D. Leddon, N. Scafetta, "The Diffusion entropy and waiting time statistics of hard x-ray solar flares," Phys. Rev. E 65, 046203 (2002). M.F. Shlesinger, J. Klafter and B.J. West, "Lévy dynamics of enhanced diffusionapplication to turbulence," Phys. Rev. Lett. 58, 1100 (1987).

[11] J. P. Bouchaud and M. Mézard, "Wealth condensation in simple model of economy," Physica A 282, 536-545 (2000).

[12] A. Drăgulescu and V. M. Yakovenko, "Statistical mechanics of money," Eur. Phys. J. B17, 723-729, (2000).

[13] A. Drăgulescu and V. M. Yakovenko, "Evidence for the exponential distribution of income in the USA," Eur. Phys. J. B20, 585-589, (2001).

[14] A. Chakraborti, B.K. Chakrabarti, "Statistical mechanics of money: how saving propensity affects its distribution," Eur. Phys. J. B17, 167 (2000).

[15] S. Ispolatov, P. L. Krapivsky and S. Redner, "Wealth distribution in asset exchange models," Eur. Phys. J. B2, 267-276, (1998).

[16] O. Malcai, O. Biham, P. Richmond and S. Solomon, "Theoretical analysis and simulations of the generalized Lotka-Volterra model," Phys. Rev. E 66, 031102 (2002).

[17] Z. Burda, D. Johnston, J. Jurkiewicz, M. Kaminski, M. A. Nowak, G. Papp and I. Zahed, "Wealth condensation in pareto macroeconomics," Phys. Rev. E 65, 026102 (2002).

[18] M. Levy, "Market Efficiency, the Pareto Wealth Distribution, and the Levy Distribution of Stock Returns," in The Economy as an Evolving Complex System III, S. Durlauf and L. Blume (Eds.), Oxford University Press, Oxford forthcoming.

[19] J. P. Bouchaud, "Power laws in economics and finance: some ideas from physics," Quantitative Finance 1, No 1 (January 2001) 105-112.

[20] D. Sornette, "Fokker-Planck equation of distributions of financial returns and power laws," Physica A 290 (1-2), 211-217 (2001).

[21] Z.-F. Huang and S. Solomon, "Stochastic Multiplicative Processes for Financial Markets," Physica A 306 412-422 (2002).

[22] K. Lindenberg and B.J. West, The Nonequilibrium Statistical Mechanics of Open and Closed Systems, VCH Publishers, New York (1990).

[23] An economic rule which states that in an efficient market, a security must have a single price, no matter how that security is created. For example, if an option can be created using two different sets of underlying securities, then the total price for each would be the same or else an arbitrage opportunity would exist. http://www.investorwords.com . About the deviation from the law of one price, see also: K.A. Froot, "The Law of One Price over 700 Years," http://www.hbs.edu, unpublished. 
[24] M. Boianovsky and V.J. Tarascio, "Mechanical inertia and economic dynamics: Pareto on business cycles," J. of the History of Economic Thought, 205 (1998).

[25] W. Feller, An Introduction to Probability Theory and Its Applications, 3rd Edition, Vol.1, John Wiley \& Sons, New York (1968).

[26] C. Tsallis, "Possible generalization of Boltzmann-Gibbs statistics," J. Stat. Phys. 52, 479 (1988).

[27] E.W. Montroll and W.W. Badger, Introduction to the Quantitative Aspects of Social Phenomena, Gordon \& Breach, New York (1974).

[28] W.W. Badger, in Mathematical Models as a Tool for the Social Sciences, Editor B.J. West, pp.87-120, Gordon \& Breach, New York (1980).

[29] R. Gibrat, Les Inégalitś Économique, Sirey, Paris (1931).

[30] J. M. Henderson and R. E. Quandt, Microeconomic theory: a mathematical approach, McGraw-Hill, USA (1971). 


\begin{tabular}{|c|c|c|c|c|}
\hline & $\mathrm{a}$ & $\mathrm{d}$ & $c$ & $\eta$ \\
\hline $\mathrm{f}=0.2$ & $3 \mathrm{e}-3 \pm 3 \mathrm{e}-4$ & $0.147 \pm 2 \mathrm{e}-3$ & $1.64 \pm 0.05$ & $1.9 \pm 0.05$ \\
\hline $\mathrm{f}=0.3$ & $5 \mathrm{e}-3 \pm 1 \mathrm{e}-3$ & $0.56 \pm 0.02$ & $0.8 \pm 0.1$ & $3.9 \pm 0.2$ \\
\hline $\mathrm{f}=0.5$ & $0.10 \pm 0.06$ & $1.9 \pm 0.06$ & $0.5 \pm 0.1$ & $6.8 \pm 0.5$ \\
\hline
\end{tabular}

Table 1

Fitting parameters of Eq. (17) for the trade-alone economy $(\mathrm{r}=0)$, see Fig. 3. The poverty index is fixed $h=0.05$.

\begin{tabular}{|c|c|c|c|c|}
\hline & $\mathrm{a}$ & $\mathrm{b}$ & $\gamma$ & $\delta$ \\
\hline $\mathrm{f} 1(\mathrm{w})$ & $6 \mathrm{e}-5 \pm 1 \mathrm{e}-5$ & $2.6 \mathrm{e}-2 \pm 2 \mathrm{e}-3$ & $2 \pm 0.1$ & $2.15 \pm 0.05$ \\
\hline $\mathrm{f} 3(\mathrm{w})$ & $5 \mathrm{e}-4 \pm 1 \mathrm{e}-4$ & $0.17 \pm 0.02$ & $5.8 \pm 0.6$ & $3.5 \pm 0.1$ \\
\hline
\end{tabular}

Table 2

Fitting parameters of Eq. (18) for the Trade-investment economy, see Fig. 5. The social and poverty index are fixed; $f=0.3$ and $h=0.05$.

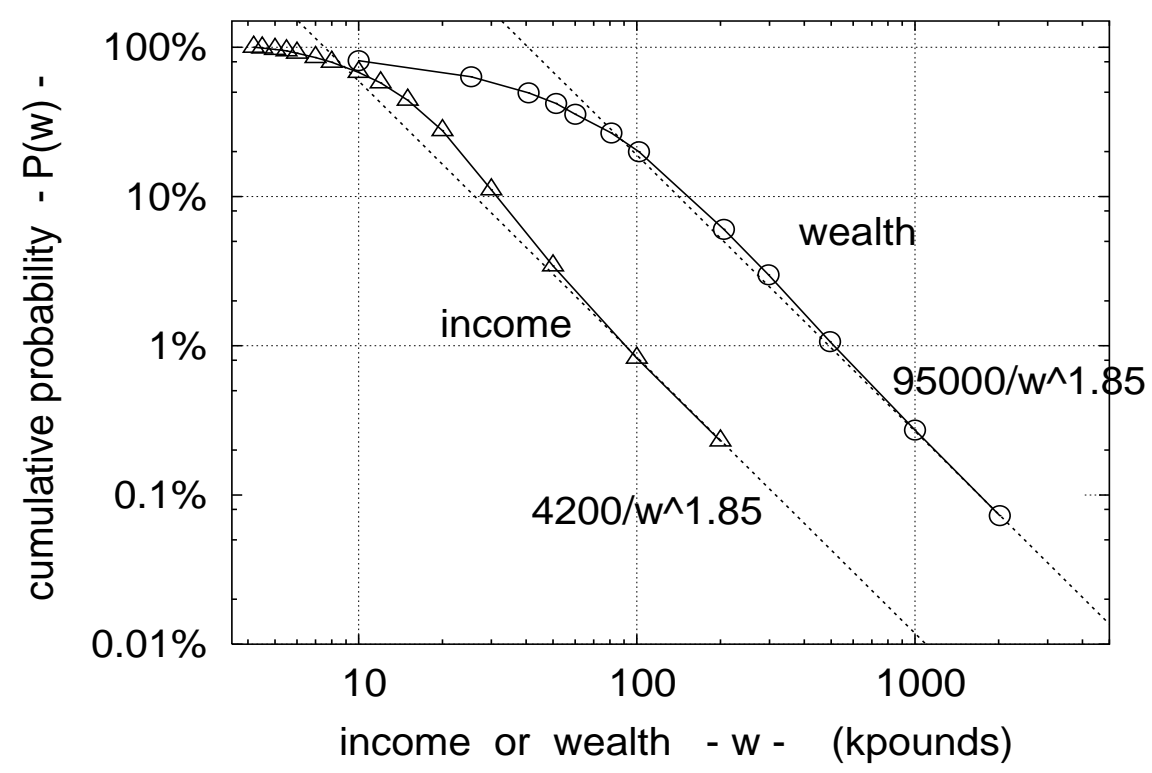

Fig. 1. Cumulative wealth (1996) and income (1998-1999) distributions in United Kingdom. 


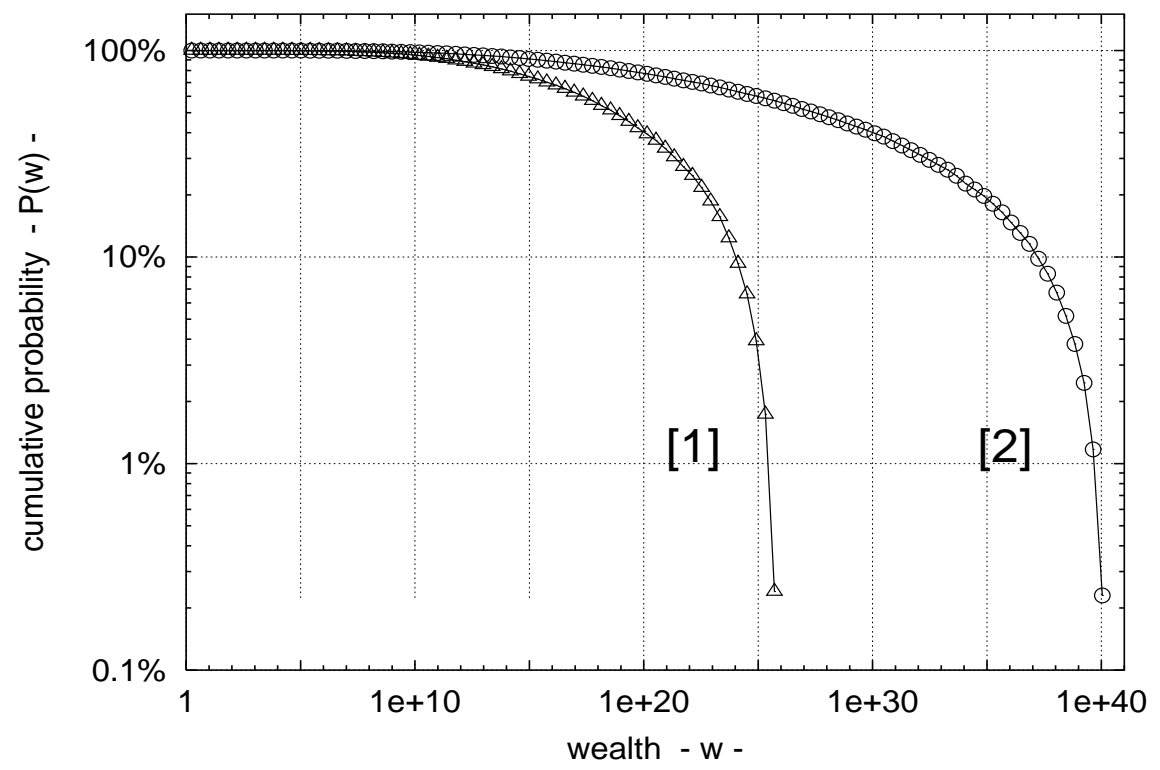

Fig. 2. Cumulative wealth distribution for the symmetry chance model. The indexes are: $h=0.05, f=0$ and $r=0$. Case [1] is after 100 million trade-interactions, and case [2] is after 200 million trade-interactions. The initial wealth distribution is uniform. The figure shows that this model yields to a huge wealth gap between the rich and poor that increases with the number of interactions. The wealth is measured in units of the poorest agent's wealth.

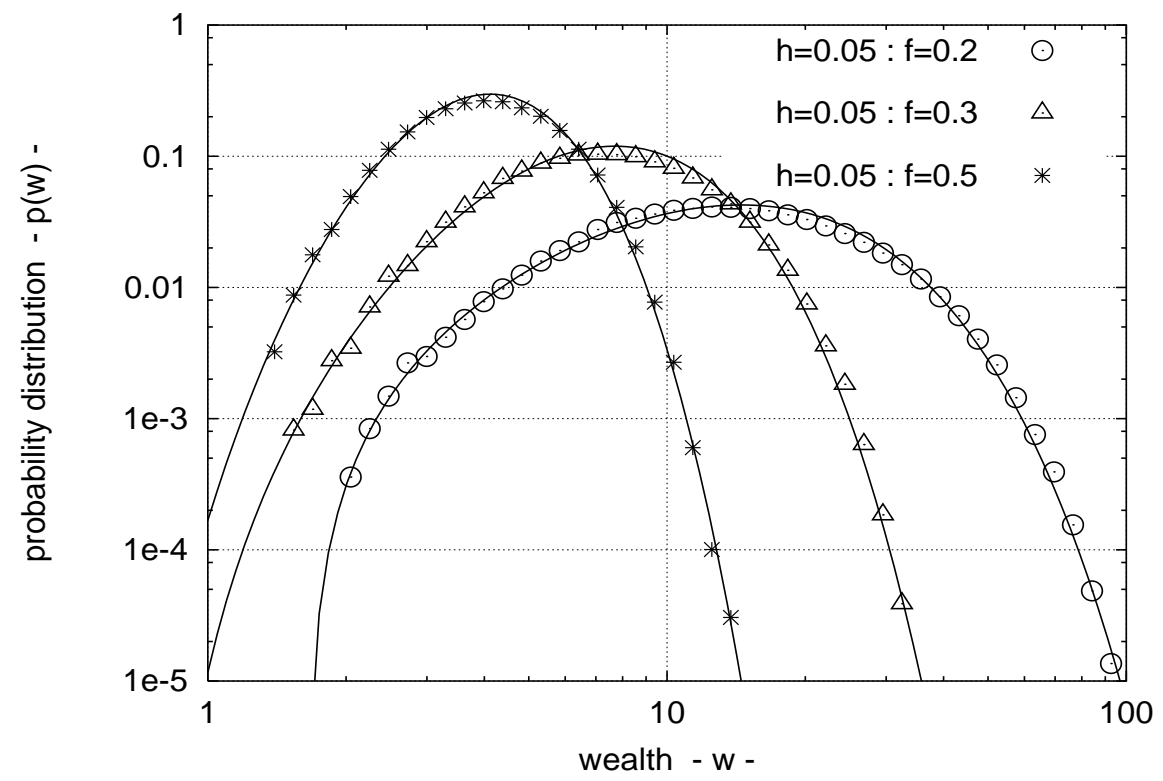

Fig. 3. Wealth probability density for the asymmetric chance model with a fixed poverty index $h=0.05$. The wealth condensation increases by decreasing the social index $f$. The investment index is $r=0$. The distributions are fitted by a Gamma distribution Eq. (17). The fitting parameters are in Table I. The wealth is in units of the poorest agent's wealth. 


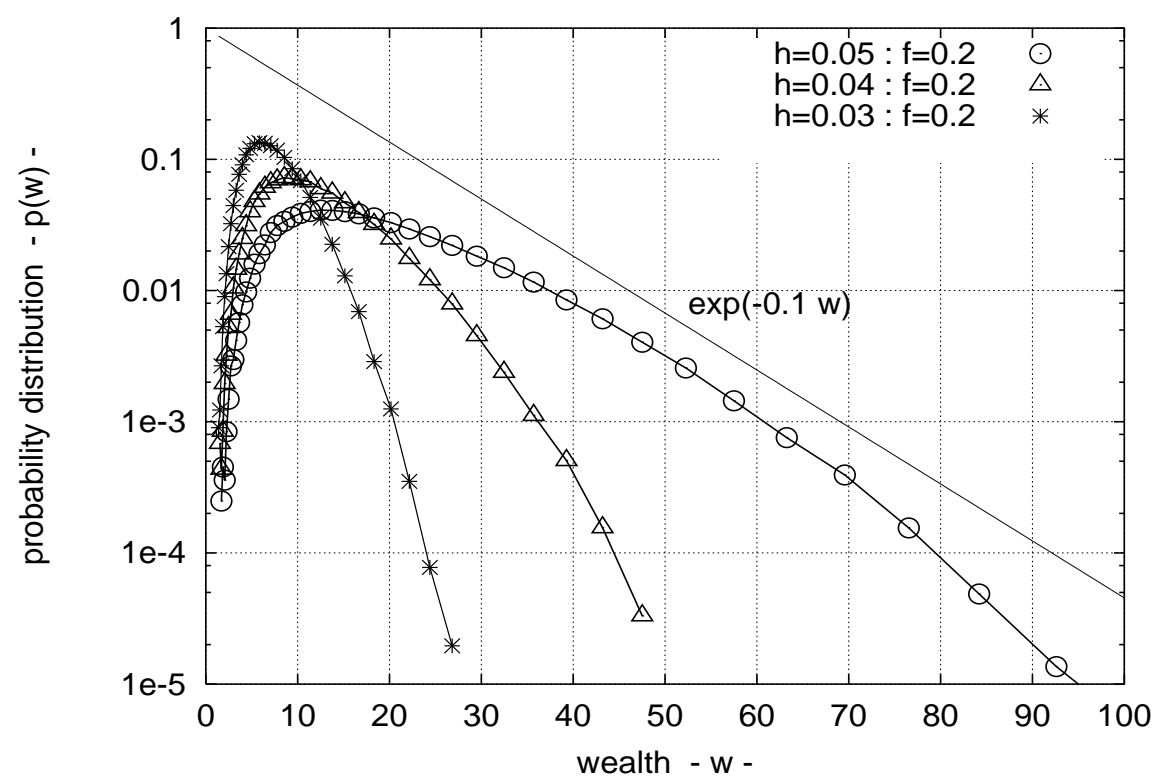

Fig. 4. Wealth probability density for the asymmetric chance model with a fixed social index $f=0.2$. The wealth condensation increases by increasing the poverty index $h$. The distributions are compared to an exponential Maxwell-Boltzmann distribution. The wealth is in units of the poorest agent's wealth.

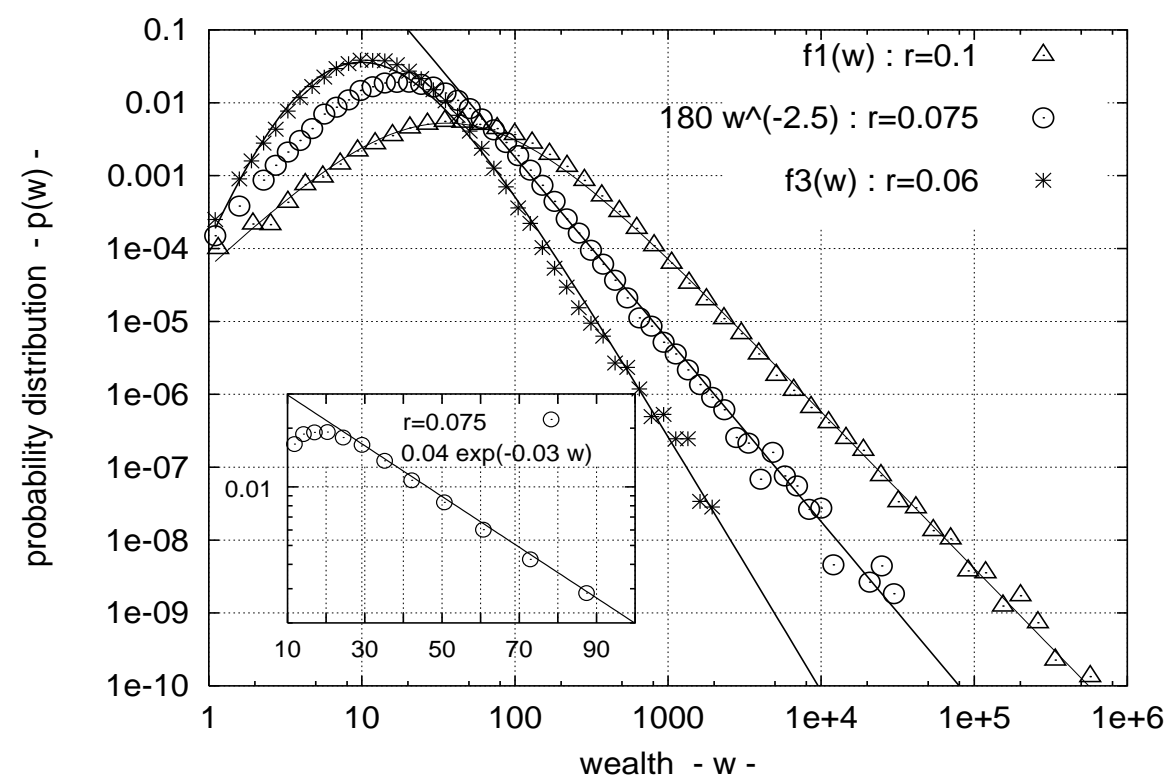

Fig. 5. Trade-investment economy. The social and poverty index are fixed; $f=0.3$ and $h=0.05$. The probability distributions (tringles) and (stars) are fitted by using Eq. (18). The fitting parameters are in Table II. The tail of the probability distribution (circles) is fitted by a power law of the type $1 / x^{\mu+1}$ where $\mu=1.5$ in the Pareto's exponent. The small picture shows the probability distribution (circles) in the interval [10:100] that is fitted with an exponential Maxwell-Boltzmann distribution. The wealth is measured in units of the poorest agent's wealth. 


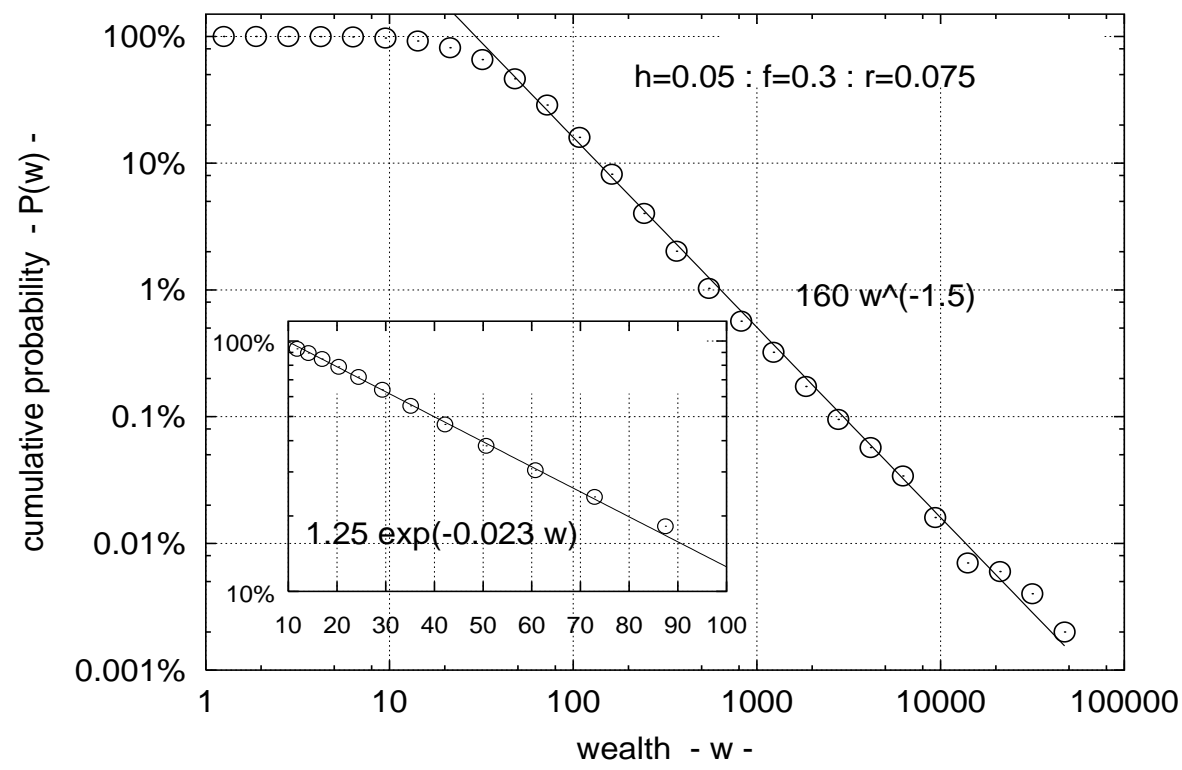

Fig. 6. Cumulative probability for an trade-investment economy with $h=0.05$, $f=0.3$ and $r=0.075$. The Pareto's exponent is $\mu=1.5 \pm 0.02$. The little picture shows that in the interval [10:100] the $\mathrm{P}(\mathrm{w})$ can be apparently fitted by an exponential Maxwell-Boltzmann distribution. The wealth is measured in units of the poorest agent's wealth.

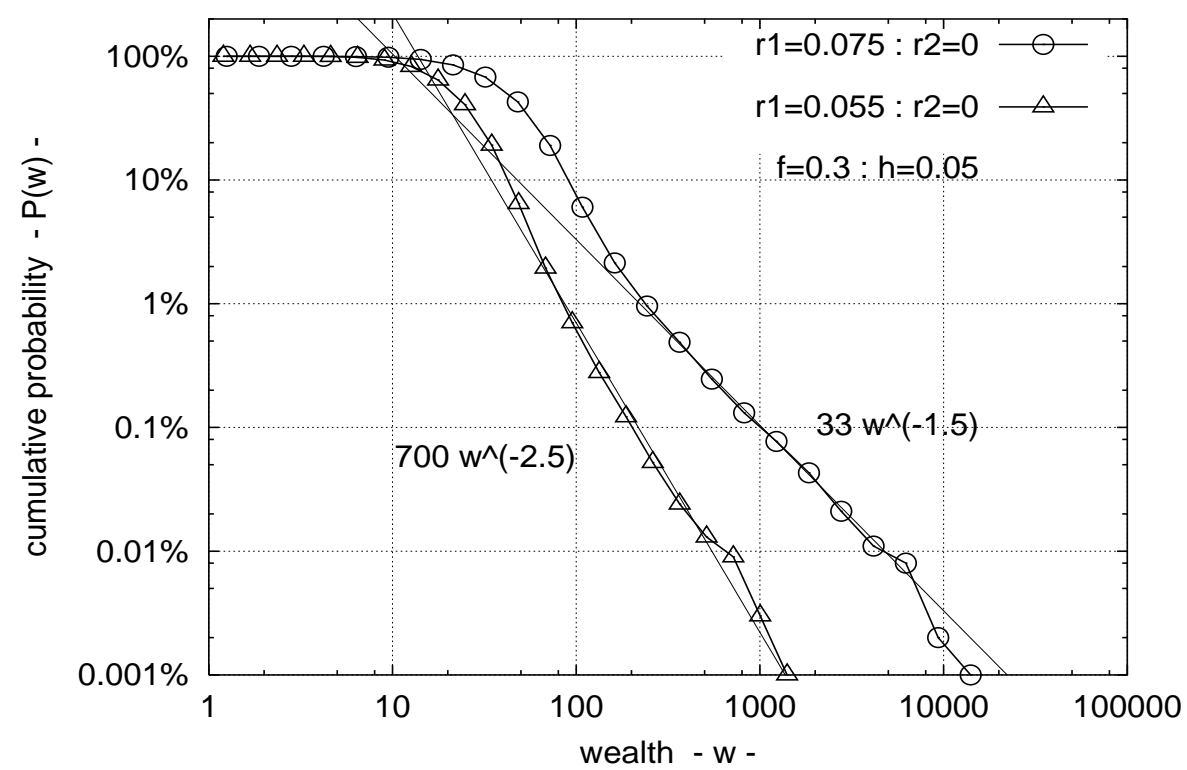

Fig. 7. Cumulative probability for a double trade-investment economy. One half of the population has the investment index $r_{2}=0$, the other half of the population has in one case $r_{1}=0.075$ and in the other $r_{1}=0.055$. In both cases the social index is $f=0.3$ and the poverty index is $h=0.05$. The Pareto's exponents are $\mu=1.5 \pm 0.02$ and $\mu=2.5 \pm 0.02$. The wealth is measured in units of the poorest agent's wealth. 


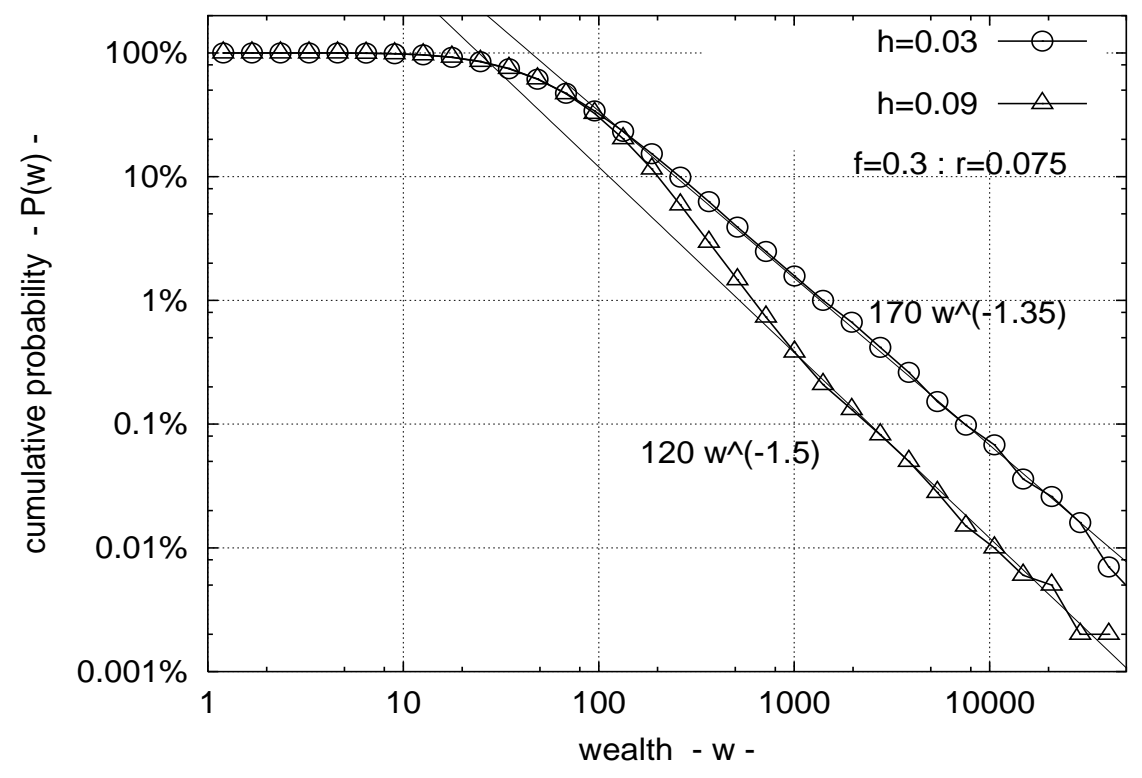

Fig. 8. Cumulative probability for two trade-investment economies with different poverty index $h$. In both cases the social index is $f=0.3$ and the investment index is $r=0.075$. The Pareto's exponents are $\mu=1.5 \pm 0.02$ and $\mu=1.35 \pm 0.02$. The wealth is measured in units of the poorest agent's wealth. 